\title{
Singlet-triplet relaxation induced by confined phonons in nanowire-based quantum dots
}

\author{
Y. Yin* \\ Hefei National Laboratory for Physical Sciences at Microscale and Department of Physics, \\ University of Science and Technology of China, Hefei, Anhui, 230026, China
}

(Dated: May 8, 2022)

\begin{abstract}
The singlet-triplet relaxation in nanowire-based quantum dots induced by confined phonons is investigated theoretically. Due to the quasi-one-dimensional nature of the confined phonons, the singlet-triplet relaxation rates exhibit multi-peaks as function of magnetic field and the relaxation rate between the singlet and the spin up triplet state is found to be enhanced at the vicinity of the singlet-triplet anti-crossing. We compare the effect of the deformation-potential coupling and the piezoelectric coupling and find that the deformation-potential coupling dominates the relaxation rates in most cases.
\end{abstract}

\section{INTRODUCTION}

As one of the most promising candidates for qubits, spins in semiconductor quantum dots (QDs) have attracted much attention in the last two decades $1-3 \mathrm{Al}$ though much progress has been made in single-electron QDs, an attractive alternative is to base the qubits on singlet-triplet (ST) states in two-electron QDs. This is mainly due to two important features of the ST states. One is the Zeeman-driven ST transition with anticrossing due to the spin-orbit coupling (SOC), which enables coherent manipulation of spin states $9-11$ The other one is the low ST relaxation rate which makes the spin polarization remains for a sufficiently long time ${ }^{12-14}$ As the QDs are either self-assembled ones sitting on the surface of the substrate or fabricated by confining electrons in quantum wells by electrodes, bulk phonons in the substrate play an important role. In low-temperature regime, acoustic bulk phonons in conjunction with the $\mathrm{SOC}$ serve as the main source of the $\mathrm{ST}$ relaxation due to the suppression of the effect of the hyperfine interaction in the presence of large magnetic field. Previous studies show that at the vicinity of the ST anti-crossing, the relaxation rate is greatly suppressed due to the mismatch of the phonon emission wave length to the dot size. Furthermore, the spin-up triplet states which couple to the singlet ground state through the SOC has a much shorter lifetime compared to the other two triplet states due to the strong ST mixing between them ${ }^{15-20}$

Despite of these progress in two-electron QDs, the complicated fabrication process makes them difficult to be scaled up, which limits their application from an industrial point of view. Recently, self-assembled twoelectron QDs embedded in InAs [111] nanowires were fabricated. ${ }^{21-23}$ The well-controlled growth process enables parallel production in massive number with similar properties, which makes these nanowire-based QDs are more suitable for integration onto silicon and scaling up for large hierarchical systems. Although similar Zeeman-driven ST transition has been obtained in nanowire-based two-electron InAs QDs, $24-26$ the ST re- laxation can be quite different due to the properties of the confined phonons in the nanowires. The nanowires are perpendicular to the substrate, making the bulk phonons in the substrate are less important than the confined phonons in nanowires. $\frac{27,28}{2}$ The regular structure of the nanowires results in the quasi-one-dimensional confined phonons, which lead to novel properties in optical absorption and transport for the nanowire-based QDs, 29,30 Therefore, these confined phonons are expected to have a pronounced impact on the ST relaxation, which has not been studied to the best of our knowledge. In this work we will investigate the ST relaxation induced by the confined phonons.

\section{MODEL AND FORMALISM}

Consider a two-electron elongate QD embedded in InAs [111] cylindrical nanowire with radius $R$ in the presence of external magnetic field $B$ along the wire. We model the QD by an anisotropic harmonic potential $V_{c}(r, z)=\frac{1}{2} m^{*} \omega_{0}^{2} r^{2}+\frac{1}{2} m^{*} \omega_{z}^{2} z^{2}$ ( corresponding to the effective dot length $d_{z}=\sqrt{\hbar \pi / m^{*} \omega_{z}}$ and dot diameter $d_{0}=\sqrt{\hbar \pi / m^{*} \omega_{0}}$ ) with $z$-axis along the wire. $m^{*}$ is the effective mass of the electron. The single-electron Hamiltonian can be expressed as

$$
H_{\mathrm{e}}=\frac{\boldsymbol{p}^{2}}{2 m^{*}}+V_{c}(r, z)+H_{\mathrm{B}}+H_{\mathrm{SO}}
$$

where $H_{\mathrm{B}}=\frac{1}{2} g \mu_{\mathrm{B}} \boldsymbol{B} \cdot \boldsymbol{\sigma}$ is the Zeeman splitting with $g$, $\mu_{\mathrm{B}}$ and $\boldsymbol{\sigma}$ being the $g$ factor of electron, Bohr magneton and Pauli matrix respectively. $H_{\mathrm{SO}}$ represents the SOC term. In this work, we concentrate on the Rashba coupling which is dominant in InAs nanowires which has the form $H_{\mathrm{SO}}=\frac{\gamma}{\hbar} \sigma^{y} p^{z}$ with $\gamma$ being the Rashba coupling strength ${ }^{24,31}$ We assume the dot diameter $d_{0} \ll d_{z}$ so that only the lowest electron subband in the radial direction is needed. We also assume the external magnetic field is weak enough so that its orbital effect can be neglected. 
The confined phonons are calculated with isotropic elastic continuum model which is widely used in the study of nanowires, carbon nanotubes and nanoparticles. ${ }^{32-37}$ The nanowire is modeled as an infinite cylinder with the stress vanishing at the surface of the wire. The displacement field of the ions induced by the confined phonons can be expressed by the quantized form (in cylindrical coordinate)

$$
\boldsymbol{u}(\boldsymbol{r})=\sum_{\nu q} \sqrt{\frac{\hbar}{2 \rho V \omega_{\nu q}}} \overline{\boldsymbol{u}}_{\nu q}(r) e^{i l \theta+i q z}\left(a_{\nu q}+a_{\nu-q}^{\dagger}\right)
$$

where $V$ is the volume of the nanowire and $\rho$ is the density. The eigenmodes $\overline{\boldsymbol{u}}_{\nu q}(r)$ and spectrum $\omega_{\nu q}$ are calculated following Refs. 38.39. Note the due to the boundary condition, generally speaking, the confined phonon modes have both longitude and transverse components.

Given the displacement field, both the deformationpotential coupling and the piezoelectric coupling can be calculated. The deformation-potential coupling is given by the divergence of the displacement field, which reads

$$
H_{\mathrm{ep}}^{\mathrm{D}}(\boldsymbol{r})=-\Xi \nabla \cdot \boldsymbol{u}(\boldsymbol{r}),
$$

where $\Xi$ is the deformation-potential coupling strength. Note that only the longitude component of the confined phonon mode has contribution to the deformationpotential coupling. The piezoelectric coupling is given by 33

$$
H_{\mathrm{ep}}^{\mathrm{P}}(\boldsymbol{r})=\frac{e}{\kappa} \int d \boldsymbol{r}_{\mathrm{e}} \frac{\nabla \cdot \boldsymbol{P}^{\mathrm{PZ}}}{\left|\boldsymbol{r}-\boldsymbol{r}_{\mathrm{e}}\right|},
$$

where $\boldsymbol{P}^{\mathrm{PZ}}$ is the polarization induced by the displacement field and $\kappa$ is the dielectric constant. For InAs [111] nanowires with wurtzite structure, $\boldsymbol{P}^{\mathrm{PZ}}$ can be expressed $\operatorname{as}^{40}$

$$
\begin{aligned}
\boldsymbol{P}^{\mathrm{PZ}}(\boldsymbol{r}) & =e_{15}\left(\partial_{r} u_{z}(\boldsymbol{r})+\partial_{z} u_{r}(\boldsymbol{r})\right) \boldsymbol{e}_{r} \\
& +\left[e_{31}\left(\partial_{r} u_{r}(\boldsymbol{r})+u_{r}(\boldsymbol{r}) / r\right)+e_{33} \partial_{z} u_{z}(\boldsymbol{r})\right] \boldsymbol{e}_{z},
\end{aligned}
$$

with $e_{15}, e_{31}$ and $e_{33}$ being the piezoelectric constants. Note that due to the anisotropy of the polarization, the piezoelectric coupling can be more sensitive to the confined phonon mode than the deformation-potential coupling.

In the following discussion, we restrict to the dilatation modes [which have angular momentum quantum number $l=0$ and angular component $\bar{u}_{\nu q}^{\theta}(r)=0$ in Eq. (2)] since only these modes can couple to the electrons in the QDs in the lowest subband of radial direction. $30,41,42$

For two-electron QDs, the total Hamiltonian of the system is given by

$$
H=\left(H_{\mathrm{e}}^{1}+H_{\mathrm{e}}^{2}+H_{\mathrm{C}}\right)+H_{\mathrm{ep}}^{1}+H_{\mathrm{ep}}^{2}+H_{\mathrm{p}},
$$

where $H_{\mathrm{C}}=\frac{e^{2}}{\kappa} \frac{1}{\left|\boldsymbol{r}_{1}-\boldsymbol{r}_{2}\right|}$ represents the Coulomb interaction between the two electrons. $H_{\mathrm{p}}=\sum_{\nu q} \hbar \omega_{\nu q} a_{\nu q}^{\dagger} a_{\nu q}$ is the Hamiltonian for the confined phonons and $H_{\mathrm{ep}}=$ $H_{\mathrm{ep}}^{\mathrm{D}}+H_{\mathrm{ep}}^{\mathrm{P}}$ is the electron-phonon coupling. The superscript " 1 " and " 2 " label the two electrons.

To construct the two-electron basis functions, it is convenient to use the separation of variables in terms of the center of mass $\boldsymbol{R}=\left(\boldsymbol{r}_{1}+\boldsymbol{r}_{2}\right) / 2$ and the relative motion $\boldsymbol{r}=\boldsymbol{r}_{1}-\boldsymbol{r}_{2}$. As the Coulomb interaction is too strong, we choose the basis function as the eigenstates of the Hamiltonian $\left(H_{\mathrm{e}}^{1}+H_{\mathrm{e}}^{2}+H_{\mathrm{C}}\right)$ without SOC and Zeeman term, which can be solved numerically by finite difference method in real space. The basis functions have the form

$$
\left\langle\boldsymbol{R}, \boldsymbol{r} \mid n_{\mathrm{R}} n_{\mathrm{r}} \eta\right\rangle=\mathcal{R}^{\mathrm{R}}(R) \mathcal{R}^{\mathrm{r}}(r) \phi_{n_{\mathrm{R}}}^{\mathrm{R}}(Z) \phi_{n_{\mathrm{r}}}^{\mathrm{r}}(z) \chi_{\eta},
$$

where $\chi_{\eta}$ represents the spin states of the two electrons, which can be expressed as

$$
\chi_{\eta}=\left\{\begin{array}{ll}
|S\rangle=\frac{1}{\sqrt{2}}(|\uparrow \downarrow\rangle-|\uparrow \downarrow\rangle), & \eta=0 \\
\left|T_{-}\right\rangle=|\downarrow \downarrow\rangle, & \eta=1 \\
\left|T_{0}\right\rangle=\frac{1}{\sqrt{2}}(|\uparrow \downarrow\rangle+|\uparrow \downarrow\rangle), & \eta=2 \\
\left|T_{+}\right\rangle=|\uparrow \uparrow\rangle, & \eta=3
\end{array} .\right.
$$

In Eq. (7), $\mathcal{R}^{\mathrm{R}}(R) / \mathcal{R}^{\mathrm{r}}(r)$ is the wave function for the lowest subband in radial direction and $\phi_{n_{\mathrm{R}}}^{\mathrm{R}}(Z) / \phi_{n_{\mathrm{r}}}^{\mathrm{r}}(z)$ is the wave function in axial direction. The subscript " $R$ " and " $r$ " label the center of mass and relative motion respectively. To guarantee the anti-symmetric of the wave function, the singlet state $|S\rangle$ always corresponds to $\phi_{n_{\mathrm{r}}}^{\mathrm{r}}(z)$ with even parity, while the triplet states $\left|T_{ \pm, 0}\right\rangle$ correspond to $\phi_{n_{\mathrm{r}}}^{\mathrm{r}}(z)$ with odd parity. The energy levels and eigenstates of the two electrons can be obtained by diagonalizing the full two-electron Hamiltonian $\left(H_{\mathrm{e}}^{1}+H_{\mathrm{e}}^{2}+H_{\mathrm{C}}\right)$ in these basis. We identify an eigenstate as singlet and/or triplet by its expectation value $\left\langle\left(\boldsymbol{\sigma}_{1}+\boldsymbol{\sigma}_{2}\right)^{2}\right\rangle$.

Treating $|i\rangle$ and $|f\rangle$ as the initial and final states, we can calculate the phonon-induced relaxation rate using Fermi's golden rule. At zero temperature, the relaxation rate induced by the confined phonons reads 43

$$
\Gamma_{f i}=\left.\sum_{j m \nu} \frac{\left|M_{\nu q_{m}}^{j}\left\langle f\left|V_{\nu q_{m}}^{j}\right| i\right\rangle\right|^{2}}{\left|\partial_{q_{m}}\left(\hbar \omega_{\nu}\left(q_{m}\right)\right)\right|} \theta(E)\right|_{\hbar \omega_{\nu}\left(q_{m}\right)=E},
$$

where $E=\left|E_{f}-E_{i}\right|$ is the energy splitting and $\theta(E)$ is the step function. $V_{\nu q}^{j}=W_{\nu q}^{j}\left(r_{1}\right) e^{i q z_{1}}+$ $W_{\nu q}^{j}\left(r_{2}\right) e^{i q z_{2}}$ comes from the total electron-phonon interaction Hamiltonian $H_{\mathrm{ep}}^{1}+H_{\mathrm{ep}}^{2}$, with $j=D$ for the deformation-potential coupling and $j=P$ for the piezoelectric coupling. The corresponding coefficients are $\left|M_{\nu q}^{\mathrm{D}}\right|^{2}=\hbar \Xi^{2} /\left(2 \pi \rho \omega_{\nu q} R^{2}\right)$ and $\left|M_{\nu q}^{\mathrm{P}}\right|^{2}=$ $16 \hbar \pi^{2} e^{2} e_{14}^{2} /\left(\kappa^{2} 2 \pi R^{2} \omega_{\nu q}\right)$. The quantity $W_{\nu q}^{\mathrm{D} / \mathrm{P}}(r)$ are given in detail in Appendix. Note that the relaxation rate induced by bulk phonons can be expressed with similar equation as Eq. (9), the main difference is that since the constant-energy surface is continuous for bulk phonons, the discrete summation over $q_{m}$ should be replaced by the integration over wave vector $\boldsymbol{q}$.

Before we represent the results, we would like to briefly summarize the three mechanisms which is crucial for the 
ST relaxation. The first one is the ST mixing. The ST relaxation favors strong ST mixing where the SOC couples the states more efficiently. The second one is the ratio between the phonon emission wave length and the QD size. The phonon emission efficiency is maximized as the two length is comparable to each other, leading to large relaxation rate. The third one is the phonon density of states (DOS) at the phonon emission energy, which is equal to the ST energy splitting. Larger DOS implies that more phonons can be emitted, which enhances the ST relaxation. The first two mechanisms manifest themselves in $\left\langle f\left|V_{\nu q_{m}}^{j}\right| i\right\rangle$ in Eq. (9), which is known as the form factor. The third one, e.g., the phonon DOS, is closely related to the quantity $\partial_{q_{m}}\left(\hbar \omega_{\nu}\left(q_{m}\right)\right)$, which is very sensitive to the phonon spectrum. Thus this one is expected to lead to pronounced differences in the ST relaxation induced by confined phonons and bulk phonons.

\section{RESULTS}

In the numerical calculation, we use the parameters for the InAs [111] wurtzite nanowires $\underline{\underline{24}-26}$ The deformationpotential strength $\Xi$ is chosen to be $5.8 \mathrm{eV}$, the density is $\rho=5900 \mathrm{~kg} / \mathrm{m}^{3}$, the static dielectric constant $\kappa$ is 15.15 , the longitude and transverse sound velocities are chosen to be $v_{L}=4410 \mathrm{~m} / \mathrm{s}$ and $v_{T}=2130 \mathrm{~m} / \mathrm{s} \mathrm{re}-$

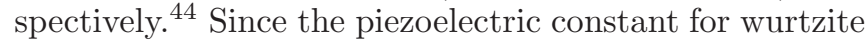
InAs nanowire is largely missing, we use the constants calculated by transformation from zinc-blende value $e_{14}$, which are $e_{15}=e_{31}=-e_{33} / 2=-e_{14} / \sqrt{3}$, with $e_{14}=3.5 \times 10^{8} \mathrm{~V} / \mathrm{m}, \stackrel{30,45}{\underline{4}}$ The $g$ factor is set to $-9.0, \underline{24}$ and the Rashba coupling constant $\gamma$ is chosen to be $1.5 \times 10^{-11} \mathrm{eV} \cdot \mathrm{m}$ (corresponding to the SO length $\lambda_{\mathrm{SO}}=$ $200 \mathrm{~nm}) \stackrel{\underline{46}}{\underline{c}}$ We set the radius of the nanowire $R$ to $15 \mathrm{~nm}$. The effective QD diameter $d_{0}$ is set to $8 \mathrm{~nm}$. The dot length $d_{z}$ is set to $70 \mathrm{~nm}$.

We employ the exact diagonalization method with the lowest 48 basis functions to converge the energy levels and ST relaxation rates $\stackrel{47.48}{=}$ The magnetic field dependence of the first four levels and the corresponding expectation value of $S^{z}=\left(\sigma_{1}^{z}+\sigma_{1}^{z}\right) / 2$ are plotted in Fig. 1(a) and (b) respectively. An anti-crossing at $B=2.36 \mathrm{~T}$ can be identified in the energy levels. The expectation values $\left\langle S^{z}\right\rangle$ show an crossing at the corresponding magnetic field, indicating a strong mixing between the singlet and spin up triplet state (ST mixing). As the orbital effect of the magnetic field is neglected, the levels show linear field dependence, which is different from the typical diskshaped QDs. 24,31 We emphasis that the mixing between the singlet and triplet states in our elongate QDs is more stronger than the QDs studied before $15,17,20$ This can be seen in the large level splitting, which is $0.14 \mathrm{meV}$ at the anti-crossing. This value is much larger than the typical value for disk-shaped GaAs QDs, which is usually a few $\mu \mathrm{eV}$. The strong mixing is not only due to the strong SOC in InAs, but also due to the large Coulomb interaction in the elongate QDs, which can enhance the relative

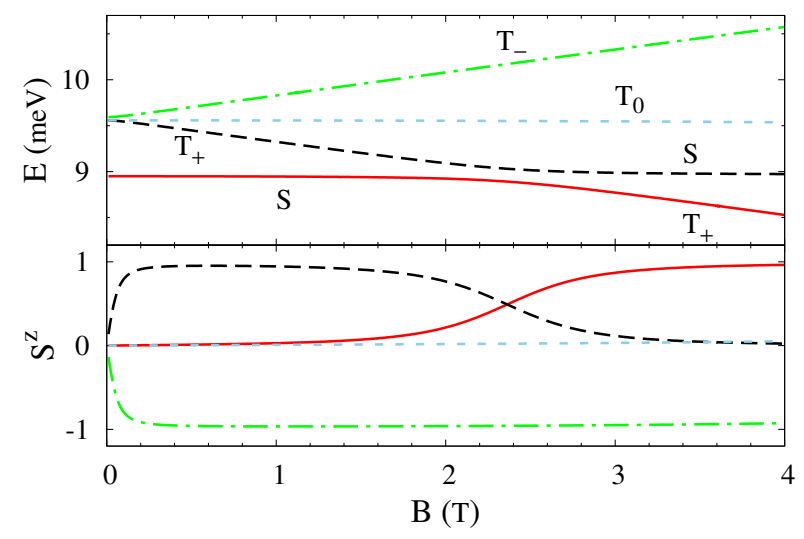

FIG. 1: (Color online) The lowest 4 energy levels and expectation value of $S^{z}=\left(\sigma_{1}^{z}+\sigma_{1}^{z}\right) / 2$ v.s. external magnetic field $B$.

strength of SOC 17,24

We start our discussion with the $\mathrm{ST}_{+}$relaxation. The magnetic field dependence of the $\mathrm{ST}_{+}$relaxation is shown in Fig. 2(a). It can be seen that the relaxation rates induced by bulk and confined phonons are very different from each other. For bulk phonons, the relaxation rate varies smoothly as a function of magnetic field. A local minimum can be found at the anti-crossing. For the confined phonons, the relaxation rate exhibits multi-peaks as a function of magnetic field. The two "sharp" peaks located at $B=1.11 \mathrm{~T}$ and $B=3.62 \mathrm{~T}$ are actually two divergent peaks. Moreover, the relaxation rate exhibits a local maximum at the anti-crossing in contrast to the local minimum for the bulk phonons. We attribute the multi-peak structure and the local maximum at the anticrossing as the two main features of the $\mathrm{ST}_{+}$relaxation rate induced by confined phonons.

The first feature, e.g., the multi-peak structure of the relaxation rate, is a direct consequence of the large DOS at the van Hove singularities in the confined phonon DOS. To see this, we plot the spectrum of the relevant confined phonon modes and the corresponding DOS in Fig. 2(b) and (c) respectively. The $\mathrm{ST}_{+}$relaxation induced by the confined phonons via the deformationpotential coupling and the piezoelectric coupling are plotted as a function of energy splitting in Fig. 2(d) [Here we only plot the relaxation rate before the anti-crossing for clarification, the relaxation after the anti-crossing has similar behavior]. It is easy to see that the peaks in the relaxation rate correspond to the van Hove singularities in the DOS, indicating the strong enhancement due to the large DOS. The divergent peaks correspond to the van Hove singularity with $q \neq 0$, while the non-divergent peaks are induced by the van Hove singularity with $q=0$. This is because as $q \rightarrow 0$, the form factor tends to zero, which will suppress the relaxation rate $\underline{\underline{43}}$ Note that the form factor can also have zeros for $q \neq 0$, which results in the dips in the relaxation rate. There are two types of zeros. One is the zeros due to the axial component (corresponding to common peaks for both the deformation- 

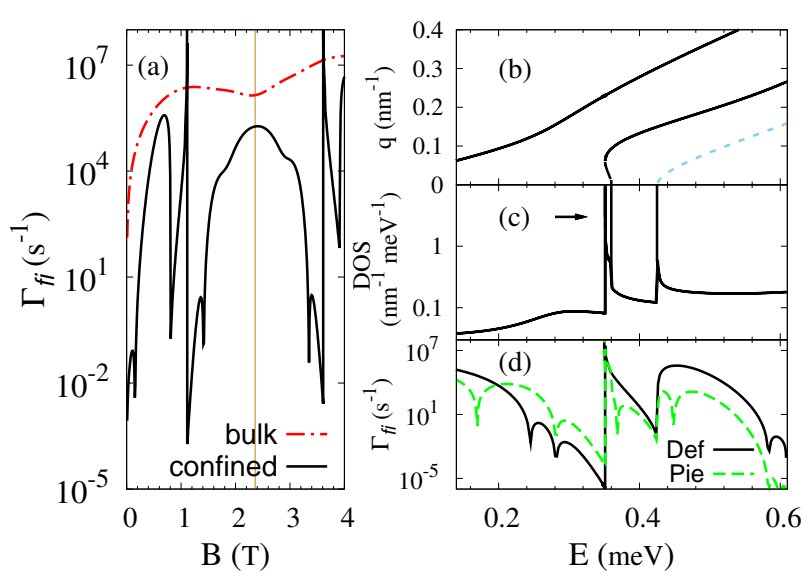

FIG. 2: (Color online) (a) $\mathrm{ST}_{+}$relaxation rate as a function of external magnetic field. The black solid/red dash-dotted curve represents the relaxation induced by confined/bulk phonons. The thin yellow line indicates the position of the anti-crossing. (b) The energy spectrum of the relevant dilatation modes. Black solid/Blue dotted curves represent the axial/radial mode as $q \rightarrow 0$. (c) The corresponding phonon DOS. The arrow indicates the van Hove singularity with phonon wave vector $q \neq 0$. (d) $\mathrm{ST}_{+}$relaxation rate induced by confined phonons as a function of energy splitting corresponding to the magnetic field region $B \in[0.0,2.36] \mathrm{T}$ (before the anti-crossing). Black solid/Green dashed curve represents the relaxation due to the deformation-potential/piezoelectric coupling.

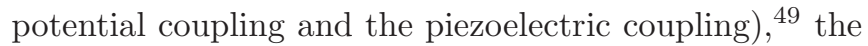
other one is the zeros due to the radial component, which induces different dips for different electron-phonon coupling mechanism. Note that the deformation-potential coupling dominates the relaxation rate in most cases except in the energy region $[0.2,0.35] \mathrm{meV}$. This is because in this region, the corresponding phonon mode tends to be transverse mode which decouples to the electrons via the deformation-potential coupling $\underline{\underline{43}}$

It can be seen for Fig. 2 that the second feature, e.g., the local maximum of the relaxation rate, occurs in the region far away from the van Hove singularities, where the confined phonon DOS exhibits a plateau. In this region, the behavior of the relaxation rate is decided by the combined effect of the ST mixing and the phonon emission efficiency. Both mechanisms enhance the relaxation rate at the anti-crossing, resulting in the local maximum. For bulk phonons, the DOS is a quadratic function of the energy splitting, so the relaxation rate can be suppressed as energy splitting decreasing, resulting in the local minimum at the anti-crossing. Note that the energy splitting $E$ here $(E=0.14 \mathrm{meV})$ is much larger than the energy splitting in typical disk-shaped GaAs QDs, so the suppression of the relaxation rate due to the decreasing of the phonon emission efficiency is absent.

To further justify the behavior of the relaxation rate at the anti-crossing, we give an estimation based on perturbation theory. We concentrate on the deformationpotential coupling since it dominates the ST relaxation rate at the vicinity of the anti-crossing. We restrict the discussion with the lowest two basis $|00 S\rangle$ and $\left|01 T_{+}\right\rangle$ with energy $\epsilon_{0}$ and $\epsilon_{1}+g \mu_{\mathrm{B}} B$ since they are almost degenerated at the vicinity of the anti-crossing. By applying the degenerated perturbation theory, we get the eigenstates

$$
\begin{aligned}
|\bar{S}\rangle & =\cos \theta|00 S\rangle+\sin \theta\left|01 T_{+}\right\rangle, \\
\left|\bar{T}_{+}\right\rangle & =-\sin \theta|00 S\rangle+\cos \theta\left|01 T_{+}\right\rangle,
\end{aligned}
$$

where $\tan \theta=t /\left(d+\sqrt{d^{2}+t^{2}}\right)$ with $t=$ $\gamma \alpha_{z}\left\langle\phi_{0}^{r}(z)\left|\partial_{z}\right| \phi_{1}^{r}(z)\right\rangle$ and $d=\left(\epsilon_{0}-\epsilon_{1}+g \mu_{\mathrm{B}} B\right) / 2$. The energy splitting between the two eigenstates is $E=2 \sqrt{d^{2}+t^{2}}$. Note that the two states are maximally mixed as $E$ reaches its minimum.

The relaxation between the singlet state $|\bar{S}\rangle$ and triplet state $\left|\bar{T}_{+}\right\rangle$can be calculated by applying Eq. 9, For bulk phonons, we have

$$
\begin{aligned}
\Gamma_{\mathrm{ST}_{+}}^{\mathrm{bulk}}= & E\left|M_{\mathrm{bulk}}^{\mathrm{D}}\right|^{2} t^{2} \int_{0}^{\pi} \sin \theta d \theta\left|I_{\mathrm{ST}_{+}}^{z}\left(q_{c} \cos \theta\right)\right|^{2} \\
& \times e^{-(5 / 4 \pi)\left(q_{c} d_{0} \sin \theta\right)^{2}}
\end{aligned}
$$

where $\left|M_{\text {bulk }}^{\mathrm{D}}\right|^{2}=\sqrt{\pi} D^{2} /\left(4 d_{z}^{3} \hbar^{4} v_{L}^{5} \rho\right)$ is a constant. $q_{c}=E /\left(\hbar v_{L}\right)$ is the bulk phonon emission wave vector. $I_{\mathrm{ST}_{+}}^{z}(q)=\left\langle 00 S\left|e^{i q z_{1}}+e^{i q z_{2}}\right| 00 S\right\rangle-\left\langle 01 T_{+}\right| e^{i q z_{1}}+$ $e^{i q z_{2}}\left|01 T_{+}\right\rangle$is the axial component of the form factor. In the situation we considered here, $I_{\mathrm{ST}_{+}}^{z}(q)$ varies slowly, so we approximate it as a constant. For small $E$, one has

$$
\Gamma_{\mathrm{ST}_{+}}^{\mathrm{bulk}} \propto E,
$$

For confined phonons, only the lowest confined phonon mode has contribution at the vicinity of the anti-crossing, so we have

$$
\begin{aligned}
\Gamma_{\mathrm{ST}_{+}}^{\mathrm{conf}}= & \left.E^{-1}\left|M_{\mathrm{conf}}^{\mathrm{D}}\right|^{2} t^{2}\left|I_{\mathrm{ST}_{+}}^{z}\left(q_{c}\right)\right|^{2} \frac{\partial q}{\partial \omega_{1 q}}\right|_{q=q_{c}} \\
& \times\left|I_{\mathrm{conf}}^{d}\left(q_{c}, \sqrt{2} d_{0}\right) I_{\mathrm{conf}}^{d}\left(q_{c}, d_{0} / \sqrt{2}\right)\right|^{2},
\end{aligned}
$$

where $\left|M_{\text {conf }}^{\mathrm{D}}\right|^{2}=D^{2} \omega_{z}^{4} /\left(2 \sqrt{\pi} d_{z} \rho R\right)$ is a constant. Note that the eigenenergy of the first confined phonon mode tends to be linear in $q$, so we can write $q_{c}=E /\left(\hbar v_{S}\right) \underline{38}$ $I_{\mathrm{ST}_{+}}^{z}(q)$ is the axial form factor which is the same as the bulk phonons since both the bulk and confined phonons can be expressed as plain wave in axial direction. $I_{\text {conf }}^{d}\left(q, d_{0}\right)=\chi_{\nu q}^{(0)}\left(k_{L} / d_{0}\right)^{2} e^{A_{L}(\nu, q)} / \sqrt{\omega_{\nu q} / \omega_{z}}$ is the radial form factor for confined phonons, with $k_{L}=\omega_{\nu q} / v_{L}$ and $A_{L}(\nu, q)=\left(q^{2}-k_{L}^{2}\right) d_{0}^{2} /(4 \pi) . \quad \chi_{\nu q}^{(0)}$ is the coefficient in the expression for the confined phonon eigenmode which is calculated numerically. It can be shown numerically that $I_{\text {conf }}^{d}\left(q, d_{0}\right)$ tends to a constant for small $q \stackrel{43}{\underline{4}}$ So for small $E$, we have

$$
\Gamma_{\mathrm{ST}_{+}}^{\mathrm{conf}} \propto E^{-1} .
$$



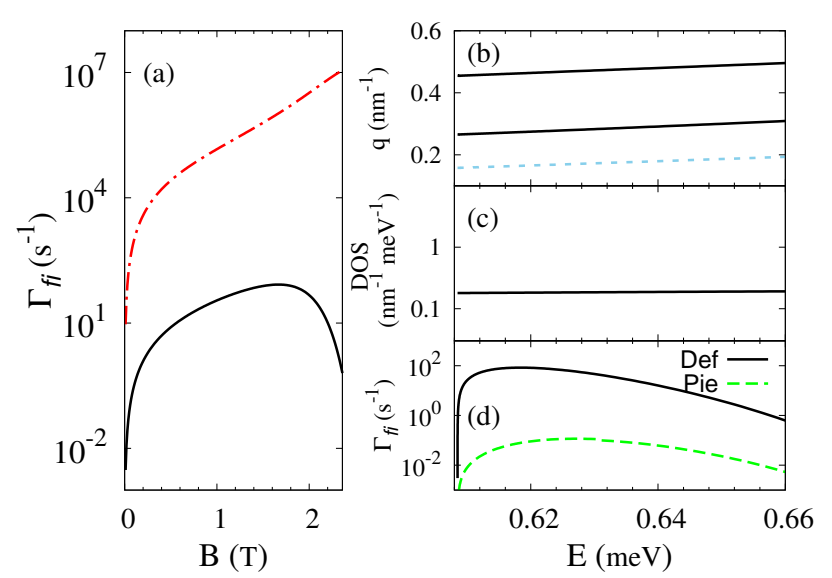

FIG. 3: (Color online) Same as Fig. 2 but for the ST - relaxation.

Comparing Eq. (13) and Eq. (15), one can see that as the energy splitting $E$ decreasing, the relaxation rate induced by bulk phonons decreases while the relaxation rate induced by confined phonons increases.

Similar features also exists for $\mathrm{ST}_{-}$and $\mathrm{ST}_{0}$ relaxation rates. The multi-peak structure in the relaxation rate due to van Hove singularities can be found for $\mathrm{ST}_{-}$ relaxation rate shown in Fig. 3. Note that the "sharp" peaks here (the first, second and the fourth peaks) are not divergent peaks since they correspond to the $q=0$ van Hove singularities. They are "sharp" since the piezoelectric coupling has a large contribution at these peaks which can be seen in Fig. 3(d). This is because these peaks are axial mode as $q \rightarrow 0$, which is favored by the piezoelectric coupling.$\underline{\underline{40}}$ Also note that for bulk phonons, the decreasing of the phonon emission efficiency due to the small phonon wave length dominates in this region. So although the DOS increasing as the magnetic field, the relaxation rate is suppressed.

In the region where the confined phonon DOS exhibits plateaus, the effect of phonon emission efficiency can be pronounced, which may suppress the relaxation rate. As an example, we compare the $\mathrm{ST}_{0}$ relaxation rate induced by bulk and confined phonons in Fig. [4. For large magnetic field, the relaxation rate is suppressed due to the decreasing of the phonon emission efficiency for confined phonons. For bulk phonons, the relaxation rate is still increasing due to the increasing of the phonon DOS. Note that the increasing of the relaxation rate for both confined phonons and bulk phonons in small magnetic field is due to the ST mixing.

\section{SUMMARY}

In summary, we have investigate the ST relaxation induced by the confined phonons in two-electron nanowirebased QDs. We find that the behavior of the relaxation rate is dominated by the large DOS at the vicinity of the van Hove singularities, while in the region where the con-
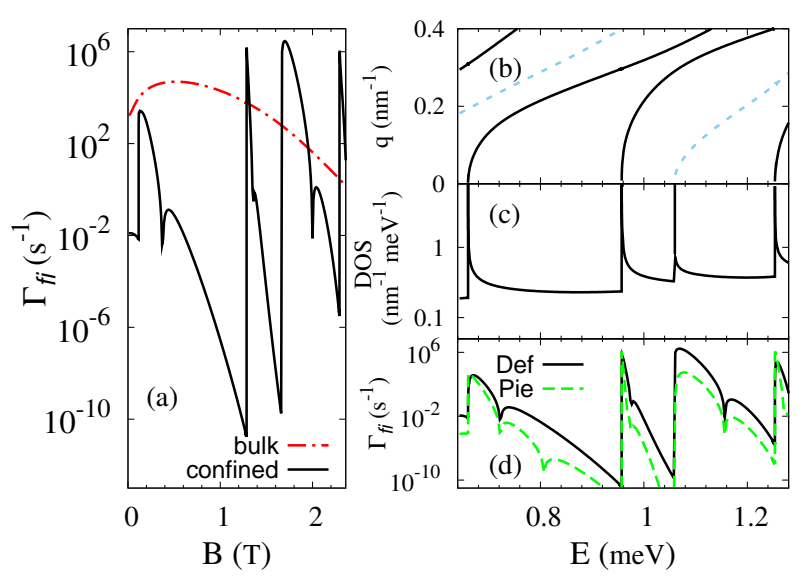

FIG. 4: (Color online) Same as Fig. 2 but for the $\mathrm{ST}_{0}$ relaxation.

fined phonon DOS is flat, the effect of the phonon emission efficiency and the ST mixing are more pronounced. This results in the multi-peak structure of the ST relaxation rate and the local maximum of $\mathrm{ST}_{+}$relaxation rate at the vicinity of the $\mathrm{ST}_{+}$anti-crossing. These features are very different from the previous results for diskshaped QDs, indicating the unique property of the confined phonons. The effect of the deformation-potential coupling and piezoelectric coupling are also discussed. We find that for InAs [111] nanowire, the deformationpotential coupling dominates the relaxation in most cases except in the region where the longitude component of the confined phonons mode is suppressed. The piezoelectric coupling is also found to be important for axial confined phonon mode at $q \rightarrow 0$. It is also worth noting that the relaxation rate induced by confined phonons is much smaller than the one induced by bulk phonons in most cases, this suggests the nanowire-based QDs are preferable for the application in quantum information and computation.

\section{Acknowledgments}

The author would like to thank M. W. Wu for proposing the topic as well as the directions during the whole investigation. This work was supported by the Natural Science Foundation of China under Grant No. 10725417, the National Basic Research Program of China under Grant No. 2006CB922005 and the Knowledge Innovation Project of Chinese Academy of Sciences and also partially by the China Postdoctoral Science Foundation.

\section{Appendix A: $W_{\nu q}^{\mathrm{D} / \mathrm{P}}(r)$ in Eq. 9}

The quantity $W_{\nu q}^{\mathrm{D} / \mathrm{P}}(r)$ is the radial component of the electron-phonon coupling $H_{\mathrm{ep}}^{\mathrm{D} / \mathrm{P}}$ with the superscript "D" for the deformation-potential coupling and "P" for the 
piezoelectric coupling. They can be obtained by substituting Eq. (2) into Eq. (3)/Eq. (4).

For the deformation-potential coupling, $W_{\nu q}^{\mathrm{D}}(r)$ is given by

$$
W_{\nu q}^{\mathrm{D}}(r)=\left(k_{L}^{2}+q^{2}\right) J_{0}\left(k_{L} r\right) \chi_{\nu q}^{(0)},
$$

where $k_{L / T}^{2}=\left(\omega_{\nu q} / v_{L / T}\right)^{2}-q^{2}$.

For the piezoelectric coupling for wurtzite InAs [111] nanowire, $W_{\nu q}^{\mathrm{P}}(r)$ is given by

$$
\begin{aligned}
W_{\nu q}^{\mathrm{P}}(r)= & \int r^{\prime} d r^{\prime} I_{0}\left(q r_{<}\right) K_{0}\left(q r_{>}\right) \\
& \times q\left[\chi_{\nu q}^{(0)} J_{0}\left(k_{L} r^{\prime}\right)\left(2 q^{2}-3 k_{L}^{2}\right)\right. \\
& \left.+\chi_{\nu q}^{(2)} J_{0}\left(k_{T} r^{\prime}\right)\left(k_{T}^{2}-4 q^{2}\right) / q^{2}\right] / \sqrt{3}
\end{aligned}
$$

where $r_{<}=\min \left(r, r^{\prime}\right)$ and $r_{>}=\max \left(r, r^{\prime}\right)$. $J_{0}(r)\left(I_{0}(r) / K_{0}(r)\right)$ is the zeroth-order (Modified) Bessel function. $\chi_{\nu q}^{(0)}$ and $\chi_{\nu q}^{(2)}$ are coefficients in the expression for the confined phonon eigenmode which are calculated numerically following Refs. 38,39.
* Electronic address: yin80@ustc.edu.cn.

1 D. Loss and D. P. DiVincenzo, Phys. Rev. A 57, 120 (1998).

2 J. M. Elzerman, R. Hanson, L. H. Willems van Beveren, B. Witkamp, L. M. K. Vandersypen, and L. P. Kouwenhoven, Nature 430, 431 (2004).

${ }^{3}$ R. Hanson, L. P. Kouwenhoven, J. R. Petta, S. Tarucha, and L. M. K. Vandersypen, Rev. Mod. Phys. 79, 1217 (2007).

4 J. R. Petta, A. C. Johnson, J. M. Taylor, E. A. Laird, A. Yacoby, M. D. Lukin, C. M. Marcus, M. P. Hanson, A. C. Gossard, Science, 309, 2180 (2005).

5 J. R. Petta, A. C. Johnson, A. Yacoby, C. M. Marcus, M. P. Hanson, and A. C. Gossard. Phys. Rev. B 72, 161301 (2005).

6 F. H. L. Koppens, C. Buizert, K. J. Tielrooij, I. T. Vink, K. C. Nowack, T. Meunier, L. P. Kouwenhoven, and L. M. K. Vandersypen, Nature 442, 766 (2006).

7 F. H. L. Koppens, J. A. Folk, J. M. Elzerman, R. Hanson, L. H. Willems van Beveren, I. T. Vink, H. P. Tranitz, W. Wegscheider, L. P. Kouwenhoven, and L. M. K. Vandersypen, Science 309, 1346 (2005).

8 C. Barthel, D. J. Reilly, C. M. Marcus, M. P. Hanson, and A. C. Gossard, Phys. Rev. Lett. 103160503 (2009).

9 J. R. Petta, H. Lu, A. C. Gossard, Science 327, 669 (2010).

10 A. C. Johnson, J. R. Petta, J. M. Taylor, A. Yacoby, M. D. Lukin, C. M. Marcus, M. P. Hanson and, A. C. Gossard, Nature 435, 925 (2005).

11 R. Hanson, L. H. Willems van Beveren, I. T. Vink, J. M. Elzerman, W. J. M. Naber, F. H. L. Koppens, L. P. Kouwenhoven, and L. M. K. Vandersypen, Phys. Rev. Lett. 94, 196802 (2005).

12 T. Fujisawa, D. G. Austing, Y. Tokura, Y. Hirayama, and S. Tarucha, Nature 419, 278 (2002).

13 S. Sasaki, T. Fujisawa, T. Hayashi, and Y. Hirayama, Phys. Rev. Lett. 95, 056803 (2005).

14 T. Meunier, I. T. Vink, L. H. Willems van Beveren, K-J. Tielrooij, R. Hanson, F. H. L. Koppens, H. P. Tranitz, W. Wegscheider, L. P. Kouwenhoven, and L. M. K. Vandersypen, Phys. Rev. Lett. 98, 126601 (2007).

15 K. Shen and M. W. Wu, Phys. Rev. B 76, 235313 (2007).
16 L. Wang, K. Shen, B. Y. Sun, and M. W. Wu, Phys. Rev. B 81, 235326 (2010).

17 V. N. Golovach, A. Khaetskii, and D. Loss, Phys. Rev. B 77, 045328 (2008).

18 M. Florescu and P. Hawrylak, Phys. Rev. B 73, 045304 (2006).

19 D. Chaney and P. A. Maksym, Phys. Rev. B 75, 035323 (2007).

20 J. I. Climente, A. Bertoni, G. Goldoni, M. Rontani, and E. Molinari, Phys. Rev. B 75, 081303(R) (2007); 76, 085305 (2007).

${ }^{21}$ M. T. Björk, B. J. Ohlsson, T. Sass, A. I. Persson, C. Thelander, M. H. Magnusson, K. Deppert, L. R. Wallenberg, and L. Samuelson, Appl. Phys. Lett. 80, 1058 (2002).

${ }^{22}$ M. T. Björk, C. Thelander, A. E. Hansen, L. E. Jensen, M. W. Larsson, L. R. Wallenberg, and L. Samuelson, Nano Lett. 4, 1621 (2004).

23 H. A. Nilsson, C. Thelander, L. E. Fröberg, J. B. Wagner, and L. Samuelson, Appl. Phys. Lett. 89, 163101 (2006).

24 C. Fasth, A. Fuhrer, L. Samuelson, V. N. Golovach, D. Loss, Phys. Rev. Lett. 98, 266801 (2007).

25 A. Pfund, I. Shorubalko, K. Ensslin, and R. Leturcq, Phys. Rev. Lett. 99, 036801 (2007).

26 A. Pfund, I. Shorubalko, K. Ensslin, and R. Leturcq, Phys. Rev. B 79, 121306(R) (2009).

27 B. J. Ohlsson, M. T. Björk, M. H. Magnusson, K. Deppert, L. Samuelson, and L. R. Wallenberg, Appl. Phys. Lett. 79, 3335 (2001).

28 H. Shtrikman, R. Popovitz-Biro, A. Kretinin, and M. Heiblum, Nano Lett. 9, 215 (2009).

29 G. Lindwall, A. Wacker, C. Weber, and A. Knorr, Phys. Rev. Lett. 99, 087401 (2007).

30 C. Weber, A. Fuhrer, C. Fasth, G. Lindwall, L. Samuelson, and A. Wacker, Phys. Rev. Lett. 104, 036801 (2010).

31 C. L. Romanoa, P. I. Tamboreneaa, and S. E. Ulloa, Physica. E, 41, 1577 (2009).

32 A. N. Cleland, Foundation of Nanomechanics (SpringerVerlag, Berlin, 2003).

33 T. Takagahara, Phys. Rev. Lett. 71, 3577 (1993).

34 H. Suzuura and T. Ando, Phys. Rev. B 65, 235412 (2002).

35 P. Chassaing, F. Demangeot, N. Combe, L. Saint-Macary, 
M. L. Kahn, and B. Chaudret, Phys. Rev. B 79, 155314 (2009).

36 S. Yu, K. W. Kim, M. A. Stroscio, and G. J. Iafrate, Phys. Rev. B 51, 4695 (1995).

37 S. M. Komirenko, K. W. Kim, M. A. Stroscio, and V. A. Kochelap, Phys. Rev. B 58, 16360 (1998).

38 B. A. Auld, Acoustic Fields and Waves in Solids (Wiley, New York, 1973).

39 M. A. Stroscio, K. W. Kim, S. Yu, and A. Ballato, J. Appl. Phys 76, 4670 (1994).

40 C. Weber and A. Wacker, phys. stat. sol. (b) 246, 337 (2009).

41 N. Nishiguchi, Phys. Rev. B 54, 1494 (1996).

42 Note that this is due to the fact that the InAs [111] nanowire is wurtzite structure. For zinc-blende nanowire, piezoelectric coupling can also couple to phonon modes with $l= \pm 2$.

43 Y. Yin and M. W. Wu, arXiv:1003.1614.

44 Semiconductors Landolt-Börnstein, edited by O. Madelung, Vol. 17a, (Springer-Verlag, Berlin, 1987).

45 A. D. Bykhovski, V. V. Kaminski, M. S. Shur, Q. C. Chen, and M. A. Khan, Appl. Phys. Lett. 68, 818 (1996).

46 A. E. Hansen, M. T. Björk, C. Fasth, C. Thelander, and L. Samuelson, Phys. Rev. B 71, 205328 (2005).

47 J. L. Cheng, M. W. Wu, and C. Lü, Phys. Rev. B 69, 115318 (2004).

48 J. H. Jiang, Y. Y. Wang, and M. W. Wu, Phys. Rev. B 77, 035323 (2008).

49 D. V. Bulaev, B. Trauzettel, and D. Loss, Phys. Rev. B 77, 235301 (2008). 


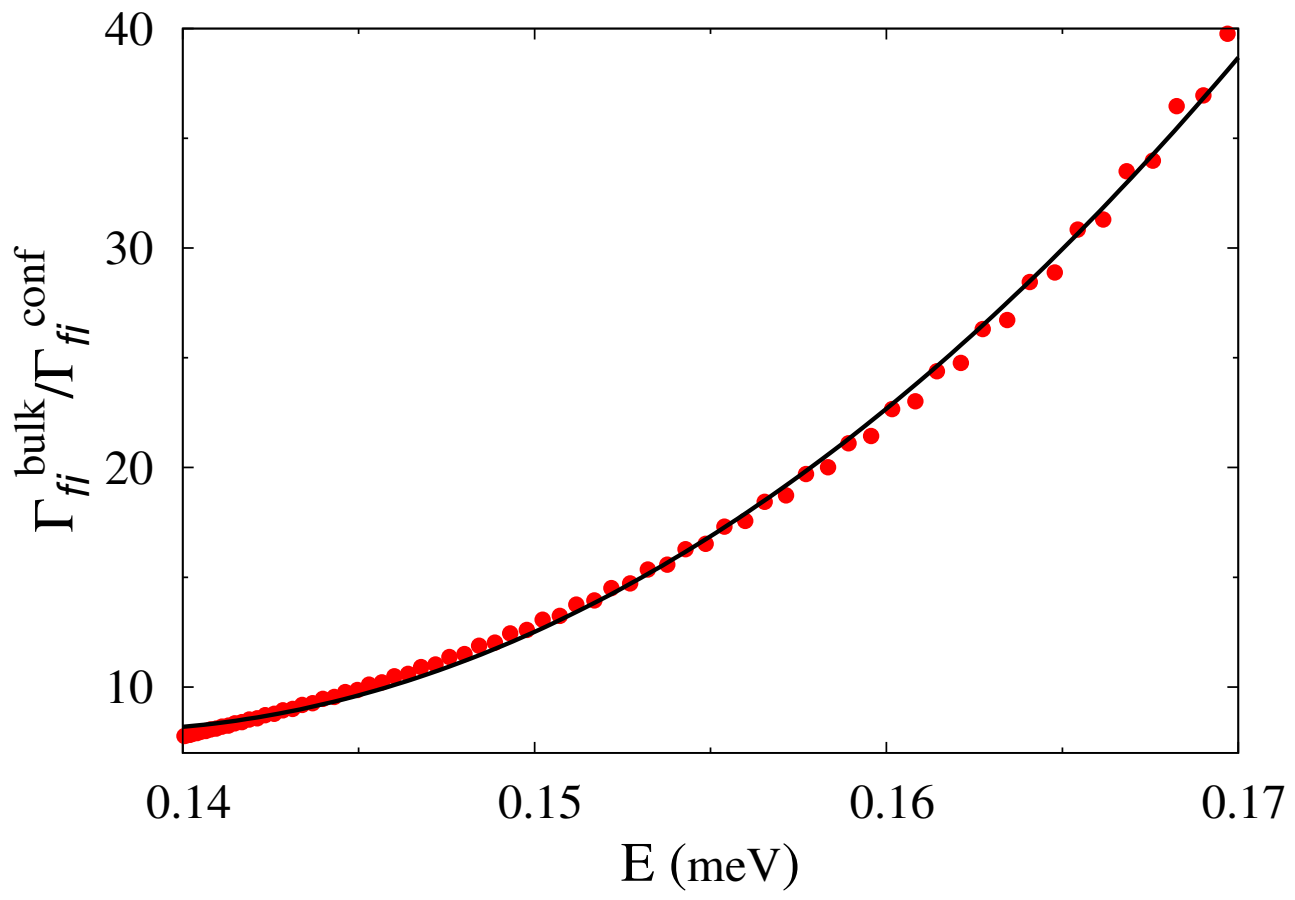

